\title{
Current perspectives on the role of TRAMP in nuclear RNA surveillance and quality control
}

This article was published in the following Dove Press journal:

Research and Reports in Biochemistry

15 April 2015

Number of times this article has been viewed

\section{Kewu Pan \\ Zhe Huang \\ Jimmy Tsz Hang Lee \\ Chi-Ming Wong}

State Key Laboratory of Pharmaceutical Biotechnology, Department of Medicine, Shenzhen Institute of Research and Innovation, the University of Hong Kong, Pokfulam, Hong Kong
Correspondence: Chi-Ming Wong State Key Laboratory of Pharmaceutical Biotechnology, Department of Medicine, Shenzhen Institute of Research and Innovation, the University of Hong Kong, L8-43, 2I Sassoon Road, Pokfulam, Hong Kong

Tel +85239179747

Fax +85228162095

Email wispwong@hku.hk
Abstract: The TRAMP complex assists the nuclear exosome to degrade a broad range of ribonucleic acid (RNA) substrates by increasing both exoribonucleolytic activity and substrate specificity. However, how the interactions between the TRAMP subunits and the components of the nuclear exosome regulate their functions in RNA degradation and substrate specificity remain unclear. This review aims to provide a summary of the recent findings on the role of the TRAMP complex in nuclear RNA degradation. The new insights from recent structural biological studies are discussed.

Keywords: TRAMP, nuclear exosome, NEXT, RNA surveillance

\section{Introduction}

TRAMP complex is one of the most well-characterized nuclear exosome cofactors, which enhances the activity and substrate specificity of the exosome. ${ }^{1}$ In Saccharomyces cerevisiae, TRAMP complex is a heterotrimeric complex consisting of a poly(A) polymerase (either Trf4p or Trf5p); a zinc-knuckle ribonucleic acid (RNA)-binding protein (either Air1p or Air2p); and an RNA helicase Mtr4p. ${ }^{2,3}$ The main function of TRAMP is to assist the nuclear exosome to degrade a large variety of RNA substrates, such as hypomodified initiator tRNA ${ }_{i}{ }^{\text {et }}$, abnormally processed ribonucleic RNAs (rRNAs), ${ }^{4-6}$ cryptic unstable transcripts (CUTs), ${ }^{7-10}$ long noncoding RNAs (lncRNAs), ${ }^{11}$ microRNAs (miRNAs), ${ }^{12,13}$ and normal by-products of RNA metabolism such as spliced-out introns, ${ }^{14,15}$ and to be involved in the many other RNA processes such as the maturation steps in precursor RNA processing and transfer RNA (tRNA) editing. ${ }^{16}$ Therefore, it is not surprising that the TRAMP complex is also directly involved in many RNA processing pathways such as splicing, ${ }^{15,17,18}$ RNA export, ${ }^{19,20}$ and heterochromatic gene silencing, ${ }^{21,22}$ and is even indirectly involved in the maintenance of genomic stability. ${ }^{9,23,24}$ This review summarizes recent findings on molecular mechanisms underlying how the TRAMP complex regulates nuclear RNA surveillance.

\section{Trf4p and Trf5p - non-canonical nuclear poly(A) polymerases}

Trf4p and Trf5p are two highly similar (45\% identity and $72 \%$ similarity) poly(A) polymerases. ${ }^{24}$ Previous studies showed that they exhibited negative genetic interaction with the DNA topoisomerase Top1p, and they were named as topoisomerase-related function (Trf) $4 p$ and Trf5p..$^{23,24}$ As a result, Trf4p and Trf5p were suggested to act as DNA polymerases that play a role in genome stability. ${ }^{25-27}$ Many different mechanisms for their contribution in genomic instability were proposed, for example, through the 
regulation of the levels of histone messenger RNA (mRNA) ${ }^{28}$ and non-protein coding RNA transcripts (ncRNAs), ${ }^{9,29}$ the R-loop-mediated transcription-associated recombination, ${ }^{30}$ and the recruitment of replication protein A. ${ }^{31}$ In subsequent research, the importance of Trf4p and Trf5p in RNA degradation intermediates were demonstrated, and the fact that their $\operatorname{poly}(\mathrm{A})$ polymerase activity is required for RNA degradation. ${ }^{32,33}$

As the TRAMP complex is composed of either Trf4p or Trf5p, two distinct TRAMP complexes (TRAMP4 and TRAMP5, named for the presence of Trf4p or Trf5p, respectively) co-exist in $S$. cerevisiae. The cellular level of Trf4p is approximately three times higher than that of Trf5p. That may be the reason why deletion of TRF5 generates no obvious phenotype, whereas loss of TRF4 leads to a slow-growth phenotype. ${ }^{28}$ As deletion of both TRF4 and TRF5 are inviable, ${ }^{24}$ it is highly possible that Trf4p and Trf5p have overlapping functions. ${ }^{5,14}$ However, recent studies demonstrated that deletion of TRF4 and TRF5 affected barely overlapping sets of substrates. ${ }^{14,32,34,35}$

Despite considerable structural similarities to the catalytic and central regions of the canonical poly(A) polymerase, both Trf4p and Trf5p lack the RNA-binding domain and rely on their RNA-binding partners, AIR proteins (Air1p or Air2p), to bind to their substrates. It was supported by chromatin immunoprecipitation analyses that the absence of Air $2 p$ impeded the recruitment of $\operatorname{Trf} 4 \mathrm{p}$ and $\operatorname{Trf5} \mathrm{p}$ to transcribing genes. ${ }^{36}$

\section{Airlp and Air2p - zinc knuckle RNA-binding proteins}

Both Air $1 p$ and Air2p were originally identified by a yeasttwo hybrid screening, as proteins physically interacting with Hmt1p. Air1p inhibits Npl3p methylation by suppressing Hmt1p's activity. ${ }^{19}$ AIR proteins were hence named accordingly as arginine methyltransferase-interacting RING finger proteins. ${ }^{19}$ A recent protein-protein interaction study demonstrated that Air $2 p$ could be methylated by Hmt $1 p$ and that the direct interaction between Npl3p and Air $2 p$ was significantly increased in the presence of active Hmt1p. ${ }^{37}$

The amino acid sequences of Air $1 p$ and Air $2 p$ are highly similar (45\% identity and 71\% similarity). They have five zinc knuckle $(\mathrm{ZnK})$ motifs that are critical for the binding to RNA and the assembly of the TRAMP-RNA complex. ${ }^{38,39}$ That exposed guanosines on RNA loops or in the singlestranded RNAs are recognized by the ZnK motifs of AIR proteins was shown by the quantitative solution-binding assay using fluorescence anisotropy experiments. ${ }^{39}$ This previous study also demonstrated that Air2p bonded to unstructured oligo(A) 15 RNA substrate with a dissociation constant in the lower micromolar range, but did not bind to oligo(dA) 15 DNA substrate. ${ }^{39}$ The function of ZnK motifs on AIR proteins is not only important for RNA binding, but also for protein-protein interaction. Based on mutation analysis and nuclear magnetic resonance titration experiments, the fourth and fifth $\mathrm{ZnK}$ motifs of Air2p are associated with the central domain of Trf4p, and the interaction is essential for TRAMP4 polyadenylation activity. ${ }^{39,40}$

Since no change in the growth rate of $S$. cerevisiae cells lacking either Air1p or Air2p was evident, while double deletion led to very severe growth defects, ${ }^{19}$ early publications $^{2,14}$ assumed that the functions of Air $1 p$ and Air2p were redundant. However, by RNA deep sequencing, distinct accumulation patterns of polyadenylated RNAs in the cells lacking either Air1p or Air2p were observed. ${ }^{41}$ The findings clearly indicated that Air $1 p$ and Air2p targeted specific transcripts for polyadenylation and degradation by the nuclear RNA exosome, resulting in profound physiology effects on the cell. ${ }^{41}$ In brief, ablation of Air $1 p$ causes the loss of the $2 \mu$ plasmid and $2 \mu$ CUTs, but ablation of Air $2 p$ decreases the levels of mRNAs encoding proteins for glucose transport and metabolism, while it elevates the levels of mRNAs involved in iron import. ${ }^{41}$

\section{Mtr4p - RNA helicase}

Mtr4p (also known as Dob1p) was identified as an RNA helicase critical for the regulation of TRAMP-mediated degradation by unwinding secondary structures of target RNAs and modulating the polyadenylation in the TRAMP complex..$^{42,43}$ The Mtr4p subunit of TRAMP was initially discovered as a protein that, when mutated, would result in an mRNA transport (mtr) defect with the accumulation of polyadenylated RNAs in the nucleus. It was then shown to be required for the nuclear exosome-dependent synthesis of 5.8S rRNA from its $7 \mathrm{~S}$ precursor. ${ }^{42}$ As Mtr4p is a member of the DExH-box RNA helicases family, Mtr4p may accelerate RNA degradation by the nuclear exosome through unwinding the RNA duplexes. ${ }^{44,45}$ In addition, it has been suggested that Mtr4p is also responsible for the proper recruitment of TRAMP-targeted RNAs to the nuclear exosome via the direct physical interaction between Mtr4p and the components of the nuclear exosome. ${ }^{29,46}$

\section{Molecular mechanism of the TRAMP complex}

The current model states that both helicase and poly(A) polymerase activities of the TRAMP complex are 
required, ${ }^{45}$ but not essential, ${ }^{46,47}$ for its function. Based on the findings from isothermal titration calorimetry, the dimerization of $\operatorname{Trf} 4 p$ and Air $2 p$ is likely required for efficient assembly with Mtr4p ${ }^{39} \operatorname{Tr} 4 \mathrm{p}$ and Air2p form the poly(A) polymerase subcomplexes first, and the N-terminal low-complexity regions of Trf4p and Air $2 p$ bind to the DExH adenosine triphosphate (ATP)ase core domain of the Mtr4p in a cooperative manner. ${ }^{48} \mathrm{~A}$ similar conserved region in the $\mathrm{N}$-terminus of $\mathrm{Trf5p}$ is required for Mtr4p interaction. ${ }^{3}$

The interactions between the subunits of the TRAMP complex are important for the coordination between helicase and polyadenylation activities of TRAMP. ${ }^{43}$ On one hand, Trf4p with Air2p stimulates the RNA helicase activity of Mtr4p through increasing its ATP affinity. ${ }^{49}$ On the other hand, Mtr $4 p$ detects the number of $3^{\prime}$ adenosines added and inhibits polyadenylation by controlling the activity of Trf4p. ${ }^{43} \mathrm{Mtr} 4 \mathrm{p}$ is also shown to bind to RNAs with adenosines tighter than other sequences. ${ }^{44,50}$ Mtr4p only fully binds when the $3^{\prime}$ extensions contain at least 5-6 nucleotides. ${ }^{49}$ Therefore, during polyadenylation, the binding affinity of Mtr4 $p$ to target substrate increases and induces slower dissociation of TRAMP from terminal adenosines. ${ }^{43}$

The model was supported by previous findings that polyadenylation by Trf5p was also markedly increased in strains lacking the RNA helicase Mtr4 $\mathrm{p}^{51}$ or with mutated Mtr $4 \mathrm{p}^{39,43}$ That may be the reason why RNAs adenylated by the TRAMP complex contain significantly shorter poly(A) tails than the canonical poly(A) polymerases..$^{52,53}$ These findings collectively suggested that the TRAMP complex polyadenylates the 3' ends of aberrant noncoding RNAs, and subsequently provides a docking site for the exosome to initiate RNA decay. ${ }^{40}$ However, in some cases, RNA degradation does not require the polyadenylation activity of TRAMP. ${ }^{14,47,54}$

\section{Targeting RNAs for degradation: recognition of aberrant sequences}

As mentioned in the "Molecular mechanism of the TRAMP complex" section, the TRAMP complex processes a broad range of RNAs produced by all three RNA polymerases (RNAP I, II and III). In the TRAMP complex, only the AIR proteins (Air1p and Air2p) have RNA-binding domains. Therefore, it is highly possible that the RNA-binding domains of Air $1 p$ and Air $2 p$ determine the substrate specificity of the TRAMP complex. ${ }^{2,40,41,51,55,56}$ The subcellular localizations of the TRAMP complexes may also contribute to the different substrate specificities of TRAMP4 and TRAMP5 complexes.
The TRAMP5 complex localizes mainly in the nucleolus, ${ }^{36,57}$ while the TRAMP4 complex is found throughout the entire nucleus in $S$. cerevisiae. ${ }^{58,59}$ That may be the reason why TRAMP5 is recruited co-transcriptionally to nucleolar surveillance machinery and is responsible for the degradation of aberrant 23S RNA. ${ }^{60,61}$

In addition, the physical binding of interacting proteins to TRAMPs controls differential substrate specificity. ${ }^{41}$ For example, TRAMP4, but not TRAMP5, is co-purified with Nrd1p and Nab3p, ${ }^{62,63}$ which are part of the Nrd1p-Nab3pSen $1 \mathrm{p}$ (NNS) complex that bind to the nuclear exosome to mediate transcription termination of non-polyadenylated RNAs. The Nrd1p-Trf4p interaction is required for this activity. ${ }^{63}$ According to the current model, Nrd1p interacts with RNAPII and Trf4p in a mutually exclusive manner. ${ }^{63}$ The C-terminal repeat domain (CTD) interacting domain (CID) of Nrd1p mediates the transition of the NNS complex from RNAPII to the Trf4p of the TRAMP complex. ${ }^{63} \mathrm{Nrd} 1 \mathrm{p}$ interacts with Trf4p and stimulates the RNA polyadenylation by the TRAMP complex. ${ }^{63}$ Hence, the dynamic interactions among RNAPII, Nrd1 $p$, and Trf4p regulate the NNS targets by promoting proper $3^{\prime}$ end formation of small nuclear RNAs (snRNAs) and small nucleolar RNAs (snoRNAs), and the degradation of unstable ncRNAs and CUTs in the nucleus. ${ }^{54,63}$

Interestingly, Airlp is shown to be co-purified with the poly(A) binding protein Hrb1p ${ }^{64}$ and Trf4p binds to the splicing factor Prp $16 p .{ }^{55}$ In addition, Hrb1p and Gbp2p genetically and physically interact with Mtr4p, and play an important role in the quality control of spliced mRNAs; whether TRAMP4 is specialized for quality control of spliced mRNAs remains to be explored.

\section{Modulation of the RNA exosome by TRAMP}

Although the nuclear exosome is an active ribonuclease in vivo, purified nuclear exosome only showed weak activity in vitro, particularly toward highly structured RNA substrates. ${ }^{2}$ The TRAMP complex increases the accessibility of the RNA 3' ends to nuclear exosome through polyadenylation. The $3^{\prime}$ oligo-adenosine tail then serves as docking for the exosome and unwinds the structured RNA. Although there is evidence demonstrating the requirement of ATP and polyadenylation of RNA substrates for the TRAMP complex to enhance the activity of the exosome, ${ }^{2,45}$ both poly(A) polymerase and the helicase activities of the TRAMP complex seem dispensable for the enhancement of RNA degradation by the exosome in vitro. ${ }^{47}$ Interestingly, one of 
the components of nuclear exosome Rrp6p plays an unexpectedly critical role in the enhancement of RNA degradation by purified exosomes via the TRAMP complex in vitro. ${ }^{47}$

Recent crystallographic analysis revealed that the N-terminal domains of Rrp6p and Rrp47p form a highly intertwined structure, which creates a groove that binds the $\mathrm{N}$-terminus of Mtr4p. ${ }^{46}$ In agreement with previous findings, ${ }^{65}$ overexpression of the core domains of Mtr4p is sufficient to suppress the growth defect of rrp6 $\Delta$ and thus restore specific $\operatorname{rrp} 6 \Delta$ RNA defects.

The TRAMP complex may enhance RNA degradation and specificity of the exosome via co-transcriptional recruitment. ${ }^{36}$ Co-transcriptional recruitment of the exosome cofactors is required to enhance the degradation of RNA substrates by the exosome. . $36,55,66$ The transcription elongation complex (THO) (which is involved in RNAPII transcription elongation and messenger ribonucleoprotein [mRNP] export) was suggested to be responsible for the maintenance of TRAMP occupancy at sites of snoRNA transcription in fission yeast. ${ }^{67}$ It is interesting to further explore whether the THO complex is also important for the recruitment of the TRAMP complex to the transcription sites for other RNA substrates.

\section{Conservation of TRAMP-mediated processes}

Components of the TRAMP complex are highly conserved from yeast to mammals. Schizosaccharomyces pombe's TRAMP complex, consisting of Cid14p, Air1p, and Mtr4p, is functionally homologous to the TRAMP complex in S. cerevisiae. ${ }^{68,69}$ Interestingly, $S$. pombe CID1 family of non-canonical poly(A) polymerases has six members (Cid1p, Cid11p, Cid12p, Cid13p, Cid14p, and Cid16p). ${ }^{70}$ As Cid14p is constitutively nucleolar and is required for polyadenylation and degradation of $S$. pombe rRNAs, Cid14p is considered as the functional orthologue of $S$. cerevisiae Trf4p and Trf5p. ${ }^{71}$ Unlike trf4-trf5 double mutants in budding yeast, S. pombe lacking CID14 is viable, though it suffers from an increased frequency of chromosome mis-segregation. ${ }^{68}$ In addition, S. pombe Cid14p is required for proper silent RNA (siRNA) generation through the RNA interference pathway. ${ }^{21,72}$

Similar to $S$. cerevisiae, the human TRAMP complex also consists of three subunits: a helicase hMtr4p, a non-canonical poly(A) polymerase hPAPD (PAP-associated domaincontaining) 5 or hPAPD7, and a $\mathrm{ZnK}$ protein hZCCHC7. ${ }^{73}$ Humans also contain two TRF homologues, hPAPD5 and hPAPD7, both of which exhibit $37 \%$ identity to the catalytic domain of $S$. cerevisiae Trf4p. Human Air1/2p orthologues of hZCCHC 7 share $35 \%$ and $34 \%$ identity to yeast Air1p and Air2p, respectively. In addition, hMtr4p (also named as SKIV2L2) exhibits 51\% total identity to yeast Mtr4p. Recent studies showed that hMtr4p precipitates contain $\mathrm{hZCCHC7p}$ and hPAPD5p, which strongly suggests that humans have functionally related yeast TRAMP complex in the processing and surveillance of rRNA, snRNA, snRNA, mRNA, and miRNA. ${ }^{38,73}$ However, in contrast to yeast Trf4p, the C-terminus of hPAPD5p contains a stretch of basic residues that is involved in binding the RNA substrate, which enables hPAPD5p to carry out its function without a separate RNAbinding subunit, such as AIR proteins in $S$. cerevisiae. ${ }^{74}$

$\mathrm{hMtr} 4 \mathrm{p}$ also forms a heterotrimer with a putative RNAbinding protein $\mathrm{hRbm} 7 \mathrm{p}$ and a zinc knuckle RNA-binding domain-containing protein hZCCHC8, known as nuclear exosome targeting (NEXT) complex. The NEXT complex contributes to the degradation of promoter upstream transcripts (PROMPTs), which are produced upstream of the promoters of actively transcribing protein-coding genes. ${ }^{73}$ The recruitment of NEXT to PROMPT regions is through NEXT's physical interaction with a cap-binding complex (CBC). The CBC-NEXT subcomplex mediates the RNAPII-derived transcription termination and suppresses the expression of PROMPTs in humans. ${ }^{75}$ Interestingly, the localization of the human TRAMP complex is only restricted to the nucleolus, and the NEXT complex is found in other parts of the nucleoplasm. ${ }^{73}$ The presence of the TRAMP complex and the NEXT complex in different subnuclear localizations suggests that compartmentalization of nuclear exosome cofactors may be necessary to cope with the more diverse transcripts.

In addition, a recent study demonstrated that the RNAbinding component of the NEXT complex, Rbm7p, could be phosphorylated by p38MAPK/MK2. ${ }^{76}$ Phosphorylated Rbm7p has lower RNA-binding capacity to nuclear non-coding RNA (such as PROMPTs) than its unphosphorylated form, and hence increases the stability of nuclear noncoding RNA for the stressdependent modulations of the noncoding transcriptome. ${ }^{76}$

\section{Conclusion and perspectives}

The advances in protein binding and structural analysis have advanced our knowledge of the molecular mechanisms of the TRAMP complex in nuclear RNA surveillance (Figure 1). However, many fundamental questions remain to be addressed. For example, how does the TRAMP complex determine the fate of different types of RNA? How does the TRAMP complex coordinate with other RNA processing events via protein-protein interactions? How is the TRAMP complex co-transcriptionally recruited, especially for the RNAPI- and 


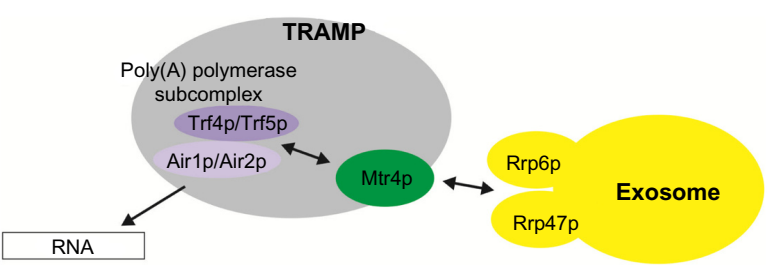

Figure I Model of interactions between RNA, TRAMP, and the exosome. RNA substrate bound by Air Ip or Air2p is oligo-adenylated by poly $(A)$ polymerase Trf4p or Trf5p until sufficient binding sites are generated for the helicase Mtr4p. Mtr4p is loosely associated with the poly $(A)$ polymerase subcomplex in the TRAMP complex. Mtr4p can only fully bind when the $3^{\prime}$ extensions contain at least 5-6 nucleotides. Therefore, during polyadenylation, the binding affinity of Mtr4p to target substrate increases. This increased affinity might contribute to Mtr4p inhibiting further oligo-adenylation and might induce slight slowing of TRAMP dissociation from the substrate. TRAMP recruits the exosome for RNA degradation through the interaction between Mtr4p and Rrp6p/Rrp47p. Mtr4p also forms a complex with other proteins, such as with Hrb Ip and Gbp2p, to mediate quality control of spliced transcripts in yeast, and with hRbm7p and hZCCHC8, as NEXT complexes to degrade PROMPTs.

Abbreviations: RNA, ribonucleic acid; NEXT, nuclear exosome targeting; PROMPT, promoter upstream transcript.

RNAPIII-derived transcripts? Can the activity and specificity of the TRAMP complex be regulated by post-translational modifications?

\section{Acknowledgments}

Special thanks to Dr Oscar Gee-Wan Wong for his critical reading of the manuscript. This work was supported by grants to CMW from the National Institutes of Health (grant number 1R01TW00829801) and the National Natural Science Foundation of China (grant number 31271361).

\section{Disclosure}

The authors report no conflicts of interest in this work.

\section{References}

1. Schmidt K, Butler JS. Nuclear RNA surveillance: role of TRAMP in controlling exosome specificity. Wiley Interdiscip Rev RNA. 2013;4: 217-231.

2. LaCava J, Houseley J, Saveanu C, et al. RNA degradation by the exosome is promoted by a nuclear polyadenylation complex. Cell. 2005;121: 713-724.

3. Losh JS, King AK, Bakelar J, et al. Interaction between the RNAdependent ATPase and poly(A) polymerase subunits of the TRAMP complex is mediated by short peptides and important for snoRNA processing. Nucleic Acids Res. Epub January 14, 2015.

4. Kadaba S, Krueger A, Trice T, Krecic AM, Hinnebusch AG, Anderson J. Nuclear surveillance and degradation of hypomodified initiator tRNAMet in S. cerevisiae. Genes Dev. 2004;18:1227-1240.

5. Kadaba S, Wang X, Anderson JT. Nuclear RNA surveillance in Saccharomyces cerevisiae: Trf4p-dependent polyadenylation of nascent hypomethylated tRNA and an aberrant form of 5S rRNA. RNA. 2006;12: 508-521.

6. Sloan KE, Bohnsack MT, Schneider C, Watkins NJ. The roles of SSU processome components and surveillance factors in the initial processing of human ribosomal RNA. RNA. 2014;20:540-550.

7. Berretta J, Pinskaya M, Morillon A. A cryptic unstable transcript mediates transcriptional trans-silencing of the Ty1 retrotransposon in S. cerevisiae. Genes Dev. 2008;22:615-626.
8. Arigo JT, Eyler DE, Carroll KL, Corden JL. Termination of cryptic unstable transcripts is directed by yeast RNA-binding proteins Nrd1 and Nab3. Mol Cell. 2006;23:841-851.

9. Frenk S, Oxley D, Houseley J. The nuclear exosome is active and important during budding yeast meiosis. PLoS One. 2014;9:e107648.

10. Loya TJ, O'Rourke TW, Reines D. A genetic screen for terminator function in yeast identifies a role for a new functional domain in termination factor Nab3. Nucleic Acids Res. 2012;40:7476-7491.

11. Beaulieu YB, Kleinman CL, Landry-Voyer AM, Majewski J, Bachand F. Polyadenylation-dependent control of long noncoding RNA expression by the poly(A)-binding protein nuclear 1. PLoS Genet. 2012;8:e1003078.

12. Boele J, Persson H, Shin JW, et al. PAPD5-mediated 3' adenylation and subsequent degradation of miR-21 is disrupted in proliferative disease. Proc Natl Acad Sci U S A. 2014;111:11467-11472.

13. Wyman SK, Knouf EC, Parkin RK, et al. Post-transcriptional generation of miRNA variants by multiple nucleotidyl transferases contributes to miRNA transcriptome complexity. Genome Res. 2011;21: 1450-1461.

14. San Paolo S, Vanacova S, Schenk L, et al. Distinct roles of noncanonical poly(A) polymerases in RNA metabolism. PLoS Genet. 2009;5:e1000555.

15. Kong KY, Tang HM, Pan K, et al. Cotranscriptional recruitment of yeast TRAMP complex to intronic sequences promotes optimal pre-mRNA splicing. Nucleic Acids Res. 2014;42:643-660.

16. Dickinson H, Tretbar S, Betat H, Morl M. The TRAMP complex shows tRNA editing activity in S. cerevisiae. Mol Biol Evol. 2012;29: 1451-1459.

17. Goebels C, Thonn A, Gonzalez-Hilarion S, et al. Introns regulate gene expression in Cryptococcus neoformans in a Pab2p dependent pathway. PLoS Genet. 2013;9:e1003686.

18. Nag A, Steitz JA. Tri-snRNP-associated proteins interact with subunits of the TRAMP and nuclear exosome complexes, linking RNA decay and pre-mRNA splicing. RNA Biol. 2012;9:334-342.

19. Inoue K, Mizuno T, Wada K, Hagiwara M. Novel RING finger proteins, Air 1p and Air2p, interact with Hmt1p and inhibit the arginine methylation of Npl3p. J Biol Chem. 2000;275:32793-32799.

20. Hackmann A, Wu H, Schneider UM, Meyer K, Jung K, Krebber H. Quality control of spliced mRNAs requires the shuttling SR proteins Gbp2 and Hrb1. Nat Commun. 2014;5:3123.

21. Bühler M, Haas W, Gygi SP and Moazed D. RNAi-dependent and -independent RNA turnover mechanisms contribute to heterochromatic gene silencing. Cell. 2007;129:707-721.

22. Houseley J, Kotovic K, El Hage A, Tollervey D. Trf4 targets ncRNAs from telomeric and rDNA spacer regions and functions in rDNA copy number control. EMBO J. 2007;26:4996-5006.

23. Sadoff BU, Heath-Pagliuso S, Castaño IB, Zhu Y, Kieff FS, Christman MF. Isolation of mutants of Saccharomyces cerevisiae requiring DNA topoisomerase I. Genetics. 1995;141:465-479.

24. Castaño IB, Heath-Pagliuso S, Sadoff BU, Fitzhugh DJ, Christman MF. A novel family of TRF (DNA topoisomerase I-related function) genes required for proper nuclear segregation. Nucleic Acids Res. 1996;24: 2404-2410.

25. Wang Z, Castaño IB, De Las Penas A, Adams C, Christman MF. Pol kappa: a DNA polymerase required for sister chromatid cohesion. Science. 2000;289:774-779.

26. Wang Z, Castaño IB, Adams C, Vu C, Fitzhugh D, Christman MF. Structure/function analysis of the Saccharomyces cerevisiae Trf4/Pol sigma DNA polymerase. Genetics. 2002;160:381-391.

27. Edwards S, Li CM, Levy DL, Brown J, Snow PM, Campbell JL. Saccharomyces cerevisiae DNA polymerase epsilon and polymerase sigma interact physically and functionally, suggesting a role for polymerase epsilon in sister chromatid cohesion. Mol Cell Biol. 2003;23: 2733-2748.

28. Reis CC, Campbell JL. Contribution of Trf $4 / 5$ and the nuclear exosome to genome stability through regulation of histone mRNA levels in Saccharomyces cerevisiae. Genetics. 2007;175:993-1010. 
29. Houseley J, Tollervey D. The nuclear RNA surveillance machinery: the link between ncRNAs and genome structure in budding yeast? Biochim Biophys Acta. 2008;1779:239-246.

30. Gavalda S, Gallardo M, Luna R, Aguilera A. R-loop mediated transcription-associated recombination in trf4Delta mutants reveals new links between RNA surveillance and genome integrity. PLoS One. 2013;8: e65541.

31. Manfrini N, Trovesi C, Wery M, et al. RNA-processing proteins regulate Mec1/ATR activation by promoting generation of RPA-coated ssDNA. EMBO Rep. 2015;16:221-231.

32. Egecioglu DE, Henras AK, Chanfreau GF. Contributions of Trf4p- and Trf5p-dependent polyadenylation to the processing and degradative functions of the yeast nuclear exosome. RNA. 2006;12:26-32.

33. Haracska L, Johnson RE, Prakash L, Prakash S. Trf4 and Trf5 proteins of Saccharomyces cerevisiae exhibit poly(A) RNA polymerase activity but no DNA polymerase activity. Mol Cell Biol. 2005;25:10183-10189.

34. Ciais D, Bohnsack MT, Tollervey D. The mRNA encoding the yeast ARE-binding protein Cth2 is generated by a novel $3^{\prime}$ processing pathway. Nucleic Acids Res. 2008;36:3075-3084.

35. Roth KM, Byam J, Fang F, Butler JS. Regulation of NAB2 mRNA $3^{\prime}$-end formation requires the core exosome and the Trf4p component of the TRAMP complex. RNA. 2009;15:1045-1058.

36. Stuparevic I, Mosrin-Huaman C, Hervouet-Coste N, Remenaric M, Rahmouni AR. Cotranscriptional recruitment of RNA exosome cofactors Rrp47p and Mpp6p and two distinct Trf-Air-Mtr4 polyadenylation (TRAMP) complexes assists the exonuclease Rrp6p in the targeting and degradation of an aberrant messenger ribonucleoprotein particle (mRNP) in yeast. $J$ Biol Chem. 2013;28831816-31829.

37. Erce MA, Abeygunawardena D, Low JK, Hart-Smith G, Wilkins MR. Interactions affected by arginine methylation in the yeast protein-protein interaction network. Mol Cell Proteomics. 2013;12:3184-3198.

38. Fasken MB, Leung SW, Banerjee A, et al. Air1 zinc knuckles 4 and 5 and a conserved IWRXY motif are critical for the function and integrity of the Trf4/5-Air1/2-Mtr4 polyadenylation (TRAMP) RNA quality control complex. J Biol Chem. 2011;286:37429-37445.

39. Holub P, Lalakova J, Cerna H, et al. Air2p is critical for the assembly and RNA-binding of the TRAMP complex and the KOW domain of Mtr4p is crucial for exosome activation. Nucleic Acids Res. 2012;40: 5679-5693.

40. Hamill S, Wolin SL, Reinisch KM. Structure and function of the polymerase core of TRAMP, a RNA surveillance complex. Proc Natl Acad Sci U SA. 2010;107:15045-15050.

41. Schmidt K, Xu Z, Mathews DH, Butler JS. Air proteins control differential TRAMP substrate specificity for nuclear RNA surveillance. RNA. 2012;18:1934-1945.

42. de la Cruz J, Kressler D, Tollervey D, Linder P. Dob1p (Mtr4p) is a putative ATP-dependent RNA helicase required for the $3^{\prime}$ end formation of 5.8S rRNA in Saccharomyces cerevisiae. EMBO J. 1998;17: $1128-1140$

43. Jia H, Wang X, Liu F, et al. The RNA helicase Mtr4p modulates polyadenylation in the TRAMP complex. Cell. 2011;145:890-901.

44. Bernstein J, Patterson DN, Wilson GM, Toth EA. Characterization of the essential activities of Saccharomyces cerevisiae Mtr4p, a 3'- $>5^{\prime}$ helicase partner of the nuclear exosome. J Biol Chem. 2008;283:4930-4942.

45. Jia H, Wang X, Anderson JT, Jankowsky E. RNA unwinding by the Trf4/Air2/Mtr4 polyadenylation (TRAMP) complex. Proc Natl Acad Sci U S A. 2012;109:7292-7297.

46. Schuch B, Feigenbutz M, Makino DL, et al. The exosome-binding factors Rrp6 and Rrp47 form a composite surface for recruiting the Mtr4 helicase. EMBO J. 2014;33:2829-2846.

47. Callahan KP, Butler JS. TRAMP complex enhances RNA degradation by the nuclear exosome component Rrp6. J Biol Chem. 2010;285: 3540-3547.

48. Falk S, Weir JR, Hentschel J, Reichelt P, Bonneau F, Conti E. The molecular architecture of the TRAMP complex reveals the organization and interplay of its two catalytic activities. Mol Cell. 2014;55: $856-867$.
49. Weir JR, Bonneau F, Hentschel J, Conti E. Structural analysis reveals the characteristic features of Mtr4, a DExH helicase involved in nuclear RNA processing and surveillance. Proc Natl Acad Sci U SA. 2010;107: 12139-12144.

50. Bernstein J, Ballin JD, Patterson DN, Wilson GM, Toth EA. Unique properties of the Mtr4p-poly(A) complex suggest a role in substrate targeting. Biochemistry. 2010;49:10357-10370.

51. Houseley J, Tollervey D. Yeast Trf5p is a nuclear poly(A) polymerase. EMBO Rep. 2006;7:205-211.

52. Keller W. No end yet to messenger RNA 3' processing! Cell. 1995;81: 829-832.

53. Grzechnik P, Kufel J. Polyadenylation linked to transcription termination directs the processing of snoRNA precursors in yeast. Mol Cell. 2008;32:247-258.

54. Thiebaut M, Kisseleva-Romanova E, Rougemaille M, Boulay J, Libri D. Transcription termination and nuclear degradation of cryptic unstable transcripts: a role for the nrd1-nab3 pathway in genome surveillance. Mol Cell. 2006;23:853-864.

55. Vanácová S, Wolf J, Martin G, et al. A new yeast poly(A) polymerase complex involved in RNA quality control. PLoS Biol. 2005;3:e189.

56. Wyers F, Rougemaille M, Badis G, et al. Cryptic pol II transcripts are degraded by a nuclear quality control pathway involving a new poly(A) polymerase. Cell. 2005;121:725-737.

57. Huh WK, Falvo JV, Gerke LC, et al. Global analysis of protein localization in budding yeast. Nature. 2003;425:686-691.

58. Anderson JT, Wang X. Nuclear RNA surveillance: no sign of substrates tailing off. Crit Rev Biochem Mol Biol. 2009;44:16-24.

59. Walowsky C, Fitzhugh DJ, Castaño IB, Ju JY, Levin NA, Christman MF. The topoisomerase-related function gene TRF4 affects cellular sensitivity to the antitumor agent camptothecin. J Biol Chem. 1999;274: 7302-7308

60. Wery M, Ruidant S, Schillewaert S, Leporé N, Lafontaine DL. The nuclear poly(A) polymerase and exosome cofactor Trf5 is recruited cotranscriptionally to nucleolar surveillance. $R N A$. 2009;15:406-419.

61. Lafontaine DL. A 'garbage can' for ribosomes: how eukaryotes degrade their ribosomes. Trends Biochem Sci. 2010;35:267-277.

62. Vasiljeva L, Buratowski S. Nrd1 interacts with the nuclear exosome for 3' processing of RNA polymerase II transcripts. Mol Cell. 2006;21: 239-248.

63. Tudek A, Porrua O, Kabzinski T, et al. Molecular basis for coordinating transcription termination with noncoding RNA degradation. Mol Cell. 2014;55:467-481.

64. Ho Y, Gruhler A, Heilbut A, et al. Systematic identification of protein complexes in Saccharomyces cerevisiae by mass spectrometry. Nature. 2002;415:180-183.

65. Klauer AA, van Hoof A. Genetic interactions suggest multiple distinct roles of the arch and core helicase domains of Mtr4 in Rrp6 and exosome function. Nucleic Acids Res. 2013;41:533-541.

66. Schneider C, Anderson JT, Tollervey D. The exosome subunit Rrp44 plays a direct role in RNA substrate recognition. Mol Cell. 2007;27:324-331.

67. Larochelle M, Lemay JF, Bachand F. The THO complex cooperates with the nuclear RNA surveillance machinery to control small nucleolar RNA expression. Nucleic Acids Res. 2012;40:10240-10253.

68. Win TZ, Draper S, Read RL, Pearce J, Norbury CJ, Wang SW. Requirement of fission yeast Cid14 in polyadenylation of rRNAs. Mol Cell Biol. 2006;26:1710-1721.

69. Keller C, Woolcock K, Hess D, Bühler M. Proteomic and functional analysis of the noncanonical poly(A) polymerase Cid14. RNA. 2010;16:1124-1129.

70. Stevenson AL, Norbury CJ. The Cid 1 family of non-canonical poly(A) polymerases. Yeast. 2006;23:991-1000.

71. Read RL, Martinho RG, Wang SW, Carr AM, Norbury CJ. Cytoplasmic poly(A) polymerases mediate cellular responses to $\mathrm{S}$ phase arrest. Proc Natl Acad Sci U S A. 2002;99:12079-12084.

72. Bühler M, Spies N, Bartel DP, Moazed D. TRAMP-mediated RNA surveillance prevents spurious entry of RNAs into the Schizosaccharomyces pombe siRNA pathway. Nat Struct Mol Biol. 2008;15:1015-1023. 
73. Lubas M, Christensen MS, Kristiansen MS, et al. Interaction profiling identifies the human nuclear exosome targeting complex. Mol Cell. 2011;43:624-637.

74. Rammelt C, Bilen B, Zavolan M, Keller W. PAPD5, a noncanonical poly(A) polymerase with an unusual RNA-binding motif. $R N A$. 2011;17:1737-1746.
75. Andersen PR, Domanski M, Kristiansen MS, et al. The human capbinding complex is functionally connected to the nuclear RNA exosome. Nat Struct Mol Biol. 2013;20:1367-1376.

76. Tiedje C, Lubas M, Tehrani M, et al. p38MAPK/MK2-mediated phosphorylation of RBM7 regulates the human nuclear exosome targeting complex. RNA. 2015;21:262-278.

\section{Publish your work in this journal}

Research and Reports in Biochemistry is an international, peer-reviewed, open access journal publishing original research, reports, reviews and commentaries on all areas of biochemistry. The manuscript management system is completely online and includes a very quick and fair peer-review system. Visit http://www.dovepress.com/testimonials.php to read real quotes from published authors. 